\title{
Genetic Counselling and Testing for Hereditary Breast and Ovarian Cancer: The Gent(le) Approach
}

\author{
M. De Vos ${ }^{*}, \#$, B. Poppe ${ }^{*}$, I. Delvaux, \\ G. Mortier, K. Claes, L. Messiaen \\ and A. De Paepe \\ Centre for Medical Genetics, University \\ Hospital Gent, Gent, Belgium \\ *equally contributing authors
}

\begin{abstract}
The counselling experience with 50 Flemish families in whom mutation analysis of the total coding region of the BRCA1 and BRCA2 gene has been initiated, is presented.

Genetic testing for breast-ovarian cancer susceptibility is offered by a multidisciplinary team. During the counselling sessions, special attention is given to comprehensible and emotionally acceptable communication of genetic information and to the psychosocial evaluation of the counselee. The limitations of molecular testing and the controversy surrounding cancer prevention strategies are also discussed.

The overall acceptance of mutation testing is high. Some of the problems encountered are inaccuracy of the reported family history, poor retrieval of the medical records of affected family members and the reluctance of many patients to inform their relatives about the possibility of being tested.
\end{abstract}

\section{INTRODUCTION}

In January 1997, mutation analysis of the complete coding sequence of the BRCA1 and BRCA2 genes was set up in the Centre for Medical Genetics in Gent and a genetic service for Hereditary Breast/Ovarian Cancer (HBOC)

\footnotetext{
\# Correspondence: A. De Paepe, Centre for Medical Genetics 0K5, University Hospital Gent, De Pintelaan 185, 9000 Gent, Belgium, Tel.: + 329240 3603, Fax: + 329240 4970, E-mail: Anne.DePaepe@rug.ac.be
}

families was developed. From the very beginning, we put the emphasis on a multidisciplinary approach to deal with diagnostic and predictive test requests, thereby using the experience we had with genetic testing for Huntington's disease.

It is our major concern to assist patients in making informed decisions regarding testing for the presence of a germline mutation that implies they are at high risk for cancer. The hallmark of the counselling sessions is the communication of medical and genetic information in a comprehensible and emotionally acceptable way, taking into account the psychosocial functioning of the test candidate and the interactions with his or her family.

Since 1997, 50 Flemish HBOC families or sporadic patients with early onset disease have been eligible for mutation testing of the BRCA1/2 genes. We present our counselling approach and discuss some of the problems we have encountered.

\section{THE COUNSELLING APPROACH}

\section{First counselling session}

The majority of our clients have been referred by their gynaecologist or oncologist, some by their general practitioner. Self-referral occurs rarely.

During the first counselling session, conducted by the clinical geneticist, the family history is evaluated and a family pedigree is constructed, based on the (mostly verbal) information that is available at that moment. Educational information about breast and ovarian cancer in general and HBOC in particular is given and the 
client's risk perception is evaluated. In addition, information on the characteristics and predictive value of the molecular test is given and positive as well as negative test results are anticipated.

Although all counselees are aware of the importance of a detailed pedigree, hearsay information about affected relatives tends to be incomplete and unreliable (case 1). Unfortunately, many patients fail to provide us with the missing data after the consultation, mainly because of reluctance to contact their relatives with respect to this delicate issue. We will shortly introduce an information sheet, to be sent to the patient prior to consultation. In this sheet we shall briefly explain the purpose of the first counselling session and emphasize the importance of a detailed pedigree.

Case 1: A 33-year old woman came to our clinic, out of concern with her risk of developing breast cancer. She told us that her 55-year old mother developed endometrial cancer at age 53 and recently was treated for breast cancer. The maternal grandmother had also developed endometrial cancer. Based on these data, we told the patient that the presence of a breast/ovarian cancer predisposing gene was unlikely in her family. After we retrieved additional data from the gynaecologist of her mother, we had to review our counselling conclusion: the mother of the patient developed an ovarian and not an endometrial cancer and there was no proof of endometrial cancer in the maternal grandmother. Based on this information (ovarian cancer at age 53 and breast cancer at age 55), mutation analysis of the BRCA1 and BRCA2 genes was started in the mother and a mutation in the BRCA1 gene was identified (E1221X).

\section{Weekly multidisciplinary team meeting}

On a weekly basis, the medical and family history from every patient who attends the genetic consultation is discussed and patients or families that fulfil our inclusion criteria for mutation analysis are selected. Our team consists of clinical geneticists, a psychologist, molecular geneticists, oncologists and gynaecologists, who all participate in the discussion.
Mutation analysis of the BRCA1 and BRCA2 genes is offered to families with at least 3 first degree relatives with breast and/or ovarian cancer, or with at least 2 first and/or second degree relatives with breast and/or ovarian cancer diagnosed before the age of 45 years [4]. Some families don't fulfil these inclusion criteria but are eligible for molecular testing because of the presence of bilateral breast cancer[7], specific anatomo-pathological features of the tumour $[1,6]$, the presence of breast and ovarian cancer in the same patient or the presence of associated tumours, such as colonic, prostate and pancreatic cancer $[5,10]$. A lack of potential female carriers in small families or an excess of males have to be taken into account in the selection procedure as well. Other family cancer syndromes have to be considered in the differential diagnosis: for instance, mutation analysis of the PTEN gene was offered to a patient who developed breast cancer and a non-Hodgkin lymphoma at age 54 [8]. Two relatives of this patient developed endometrial carcinoma.

The psychological profile of the test candidates should also be taken into account: asymptomatic women who feel extremely worried about the fact that they might have a 50\% risk of carrying a deleterious mutation, but whose affected relatives have already died, can also be eligible for a (predictive) molecular test. Although in such instances a risk reduction cannot be established if no mutation is found, we believe that offering the test should be considered in those patients, in order to achieve maximal psychological support and an optimal diagnostic work up.

A family tailored approach is crucial in the final decision of whether or not mutation testing is offered. With respect to this, close contact with the referring physicians, as well with the physicians of the affected relatives, is indispensable. As it appears to be impossible to verify the diagnosis in all affected individuals (with their consent), the decision whether to initiate testing is largely based on hearsay data. An important drawback of this strategy is the risk of initiating the molecular analysis in families who eventually turn out not to have a convincing cancer history after all. However, in 
order to avoid delay in the molecular analysis, we don't postpone starting with the test until all clinical information from relatives is obtained. On the other hand we review our initial decision to decline molecular testing whenever additional information is indicative for the presence of a hereditary predisposition. Although we try hard to retrieve all necessary medical information, our efforts are sometimes unsuccessful.

The overall acceptance of mutation testing after the first counselling session is high: so far, all patients who received genetic counselling and whose personal or family history was indicative of a genetic predisposition for breast/ovarian cancer, consented, to blood sample collection for a diagnostic molecular analysis of the BRCA1 and BRCA2 genes.

\section{The predictive test protocol}

If a mutation is identified in a patient, his or her relatives are eligible for a predictive test from the age of 18 years. In the predictive test setting, a decision counselling session is provided after the intake session. This session is conducted by the psychologist and includes a battery of psychometric tests in order to evaluate the anxiety and depression level of the test candidates, as well as an interview focusing on the social support and coping mechanisms. If the test candidate is ready to proceed, the blood sample is taken.

Out of the 16 patients who requested predictive testing, only one patient declined predictive mutation testing after the first counselling session. She said she expected more mental benefit from a regular cancer-screening programme than from certainty about her carrier status.

One of the pitfalls we encountered in predictive testing, is family pressure, which may hamper the principle of free decision making and which should be anticipated on (case 2).

Case 2: A 29-year old woman requested predictive mutation testing after a mutation in the BRCA1 gene was identified in her mother. The 53-year old mother became depressed a few months after her treatment for breast cancer and was emotionally not ready to receive her test results. She refused permission to use her test results for molecular testing in her daughter. After a discussion with her mother, the daughter had changed her point of view and told us that she no longer wanted to proceed with testing, because of fear of her own emotional response to a positive test result.

In the first step of the molecular analysis, which takes approximately 3 months, exon 11 of the BRCA1 gene and exons 10 and 11 of the BRCA2 gene are screened with the protein truncation test [3].

In addition, PCR analysis is performed in order to detect the Dutch founder mutations caused by genomic deletions encompassing exons 13 and 22 [9] and to detect the $6.4 \mathrm{~kb}$ duplication encompassing exon 13 of the BRCA1 gene [11].

After this first step, a letter is sent to all family members who participated in DNA testing. This letter informs them about the availability of results and invites them to discuss the results at a second counselling session.

\section{Second counselling session}

The purpose of this second session is to assess the knowledge of the test candidates and their emotional attitude towards the information they received three months earlier. The family history is also reassessed in order to obtain optimal medical data and to have an idea of the family communication process. This session is followed by a meeting with all team members, in order to re-evaluate the test request.

If no mutation was identified with the first analysis, heteroduplex analysis for all remaining exons and splicing boundaries of both genes is performed. After this second analysis, which takes approximately six months, the patients are re-invited to a final discussion of the results.

\section{Final counselling session}

The results are communicated by the clinical geneticist and the psychologist. Particular 
attention is paid to the limitations of the test, in cases where no mutation has been found.

If a mutation has been identified, additional sessions are provided by the psychologist, who contacts the patients by telephone after 1 week and after 1, 6 and 12 months.

A cardinal issue in the final counselling session is the discussion of the management options towards cancer prevention. The benefits and limitations of lifetime surveillance and prophylactic surgery are discussed with the patient. With respect to cancer surveillance, the absence of evidence based guidelines is very frustrating for the patient as well as for the geneticist. In evaluating the patients' current surveillance practices, we notice that most HBOC patients and their at risk relatives don't undergo regular ovarian ultrasound examinations. Despite the cancer prevention guidelines of the public health services [2], self-breast examination is equally uncommon.

\section{CONCLUSIONS}

When evaluating our experience of more than two years' genetic counselling and testing for HBOC, we conclude that informing test candidates, supporting them to communicate genetic information with their family and understanding their psychosocial functioning are cardinal issues in a cancer genetics clinic. We observe high acceptance of the genetic information provided, which, at least partly, can be ascribed to our counselling strategy. Especially in the predictive setting, careful psychometric evaluation and intense psychological counselling and support are indispensable.

The confirmation of the anatomopathological diagnosis seems recommendable in selected patients, since these data can shed a different light on the decision whether or not to start molecular testing. With respect to this, close contact with and adequate feedback from the referring clinicians is essential for good genetic practice.
In order to standardise test results and facilitate communication between genetic centres, the establishment of uniform inclusion criteria for molecular testing is warranted.

Fundamental and clinical research concerning prophylactic therapeutic strategies and screening regimens in high-risk individuals deserve further attention, since their potential benefit in these patients is still ill-defined.

\section{Acknowledgements}

Supported by a grant of the University of Gent (G.O.A. 12051397) to K.C. and by a grant of the Fund for Scientific Research (F.W.O.Vlaanderen) to B.P.

\section{References}

[1] Armes, J., Matthew Egan, A., Southey, M., Dite, G., McCredie, M., Giles, G., Hopper, J. and Venter, D. The histologic phenotypes of breast carcinoma occurring before age 40 years in women with and without BRCA1 or BRCA2 germline mutations. Cancer 83, (1998) 23352345.

[2] Belgische federatie tegen kanker V.Z.W., Borstkanker: cijfers, oorzaken en wat ertegen doen, 1999.

[3] K. Claes, E. Machackova, T. Callens, G. Vandercruyssen and L. Messiaen, Improved conditions for PTT analysis on BRCA1, BRCA2, NF1 and APC genes, Elseviers Trends Journal (1998), Technical Tips Online, t01457 (http://tto.trends.com).

[4] Claes, K., Machackova, E., De Vos, M., Mortier, G., De Paepe, A. and Messiaen, L. Mutation analysis of the BRCA1 and BRCA2 genes results in the identification of novel and recurrent mutations in 6/16 Flemish families with breast and/or ovarian cancer but not in 12 sporadic patients with early-onset disease. Hum. Mutat. 13, (1999) 256.

[5] Ford, D., Easton, D., Bishop, D., Narod, S. and Goldgar, D. Breast Cancer Linkage Consortium: risk of cancer in BRCAl mutation carriers. Lancet 343, (1994) 692-695.

[6] Lakhani, S., Jacquemier, J., Sloane, J., Gusterson, B., Anderson, T., van de Vijver, M. et al. 
Multifactorial analysis of differences between sporadic breast cancers and cancers involving BRCA1 and BRCA2 mutations. J. Nat. Cancer Inst. 90, (1998) 1138-1145.

[7] Ligtenberg, M., Hogervorst, F., Willems, H., Arts, P., Brink, G., Hageman, S. et al. Characteristics of small breast and/or ovarian cancer families with germline mutations in BRCA1 and BRCA2. Br. J. Cancer 79, (1999) 1475-1478.

[8] Nakahara, Y., Nagai, H., Kinoshita, T., Uchida, T., Hanato, S., Murate, T. and Saito, H. Mutational analysis of the PTEN/MMACl gene in non-Hodgkin's lymphoma. Leukemia 12, (1998) 1277-1280.

[9] Peelen, T., van Vliet, M., Petrij-Bosch, A.,
Mieremet, R., Szabo, C., van den Ouweland, A. et al. A high proportion of novel mutations in BRCAl with strong founder effects among Dutch and Belgian hereditary breast and ovarian cancer families. Am. J. Hum. Gen. 60, (1997) 10411049.

[10] Phelan, C., Lancaster, J., Tonin, P., Gumbs, C., Cochran, C., Carter, R. et al. Mutation analysis of the BRCA2 gene in 49-site specific breast cancer families. Nat. Genet. 13, (1996) 120-122.

[11] Puget, N., Sinilnikova, O., Stoppa-Lyonnet, D., Audoynaud, C., Pages, S., Lynch, H. et al. An Alu-mediated $6 \mathrm{~kb}$ duplication in the BRCAl gene: a new founder mutation? Am. J. Hum. Genet. 64, (1999) 300-302. 


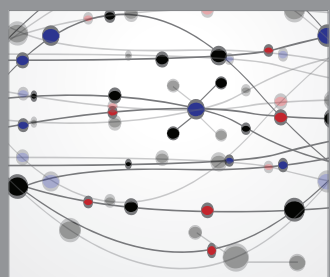

The Scientific World Journal
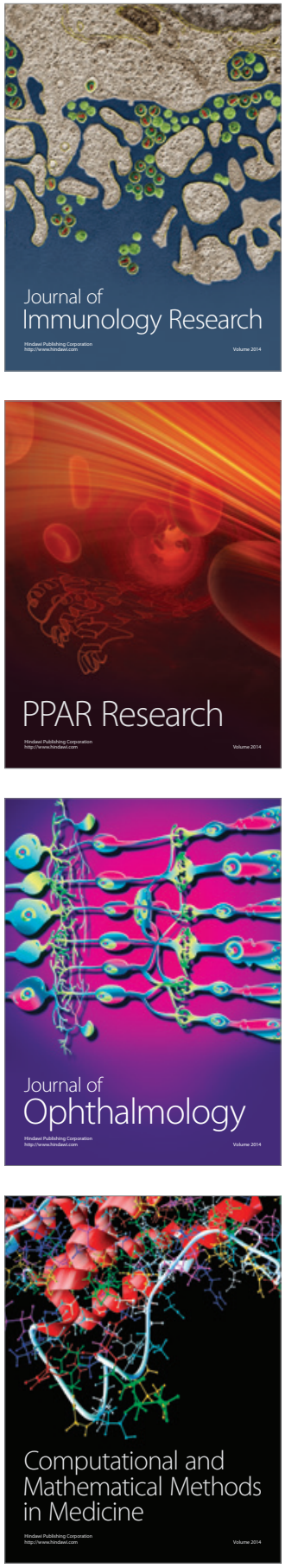

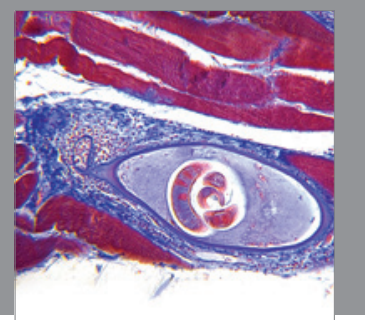

Gastroenterology

Research and Practice
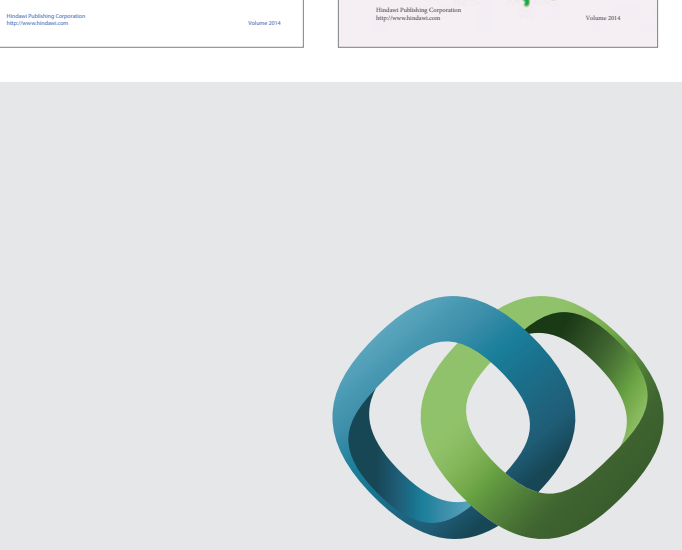

\section{Hindawi}

Submit your manuscripts at

http://www.hindawi.com
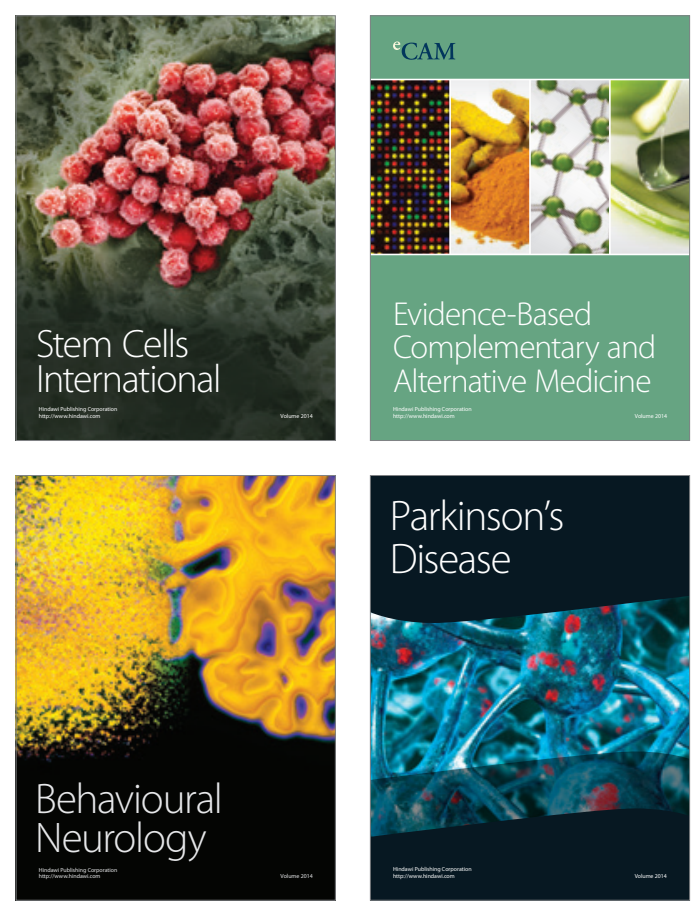

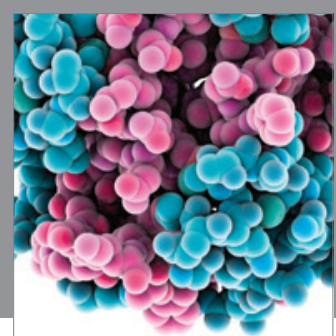

Journal of
Diabetes Research

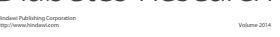

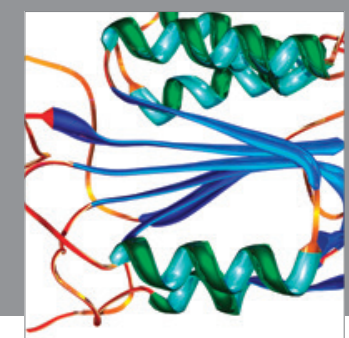

Disease Markers
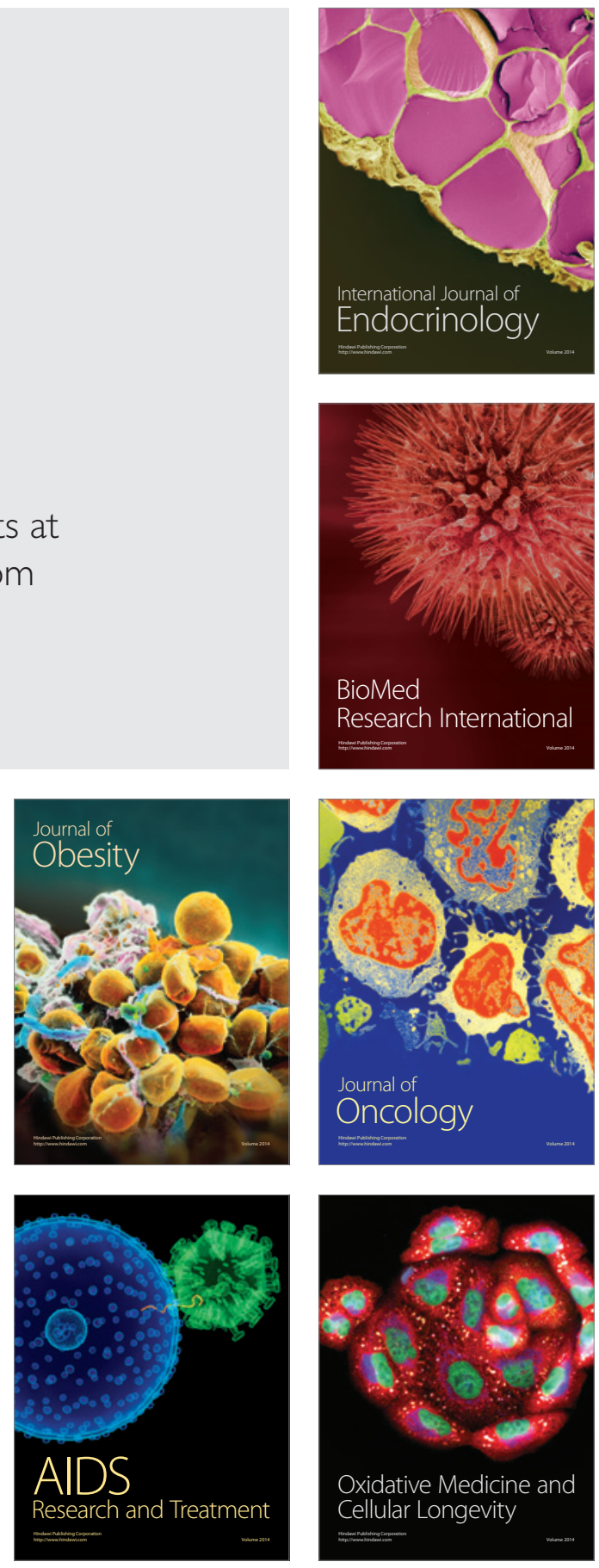